\title{
DIE ANFÄNGE DER SPANISCHEN MUSIKWISSENSCHAFT AUS DEM BLICKWINKEL DER DEUTSCHSPRACHIGEN KOLLEGENSCHAFT. EINE KLEINE SPURENLESE ZU FELIPE PEDRELL UND HIGINIO ANGLÉS
}

\author{
THE BEGINNINGS OF SPANISH MUSICOLOGY FROM THE PERSPECTIVE OF \\ GERMAN-SPEAKING COLLEAGUES. A SMALL TRAIL TO FELIPE PEDRELL \\ AND HIGINIO ANGLÉS
}

\section{LOS INICIOS DE LA MUSICOLOGÍA ESPAÑOLA DESDE LA PERSPECTIVA DE LOS COLEGAS DE HABLA ALEMANA. UN PEQUEÑO SENDERO A FELIPE PEDRELL Y HIGINIO ANGLÉS}

\author{
Thomas Hochradner \\ Universität Mozarteum Salzburg (Austria) \\ thomas.hochradner@moz.ac.at \\ ORCID iD: http://orcid.org/0000-0003-1536-5785
}

Francesc Bonastre i Bertran in memoriam

\begin{abstract}
Zusammenfassung
Felipe Pedrell und Higinio Anglés, beide gebürtige Katalanen, sind durch ihre Verbindungen zur deutschsprachigen Musikwissenschaft in ihren musikologischen Studien nachhaltig beeinflusst worden. Einesteils wird so den Anfängen der Musikwissenschaft in Spanien mit der Weg gewiesen, andernteils brachten ihre Leistungen - vor allem Editionen, aber auch Schriften - dem deutschsprachigen Raum erstmals Kenntnis über die Musik in Spanien besonders des Mittelalters und der Renaissance. Wertschätzung erfuhren sie nicht nur durch Kontakte mit befreundeten Fachkollegen, sondern auch durch eine fortwährende Präsenz in der deutschsprachigen Musiklexikographie.
\end{abstract}

\section{Schlüsselwörter}

Felipe Pedrell - Higinio Anglés - Kontakte zur deutschsprachigen Musikwissenschaft - Rezeption ihrer Schriften im deutschsprachigen Raum.

\footnotetext{
Abstract

Felipe Pedrell and Higinio Anglés, both Catalans by birth, were strongly infl uenced by their contact to musicologists in German-speaking countries. On the one hand, thus their studies have been paved the way, on the other hand their achievements mostly the musical editions, but also certain writings - gave a first impression of Spains' music history especially within the Medieval
}

Ages and in times of the Renaissance. This was estimated by their German-speaking colleagues' appreciation, as well as by a lasting presence of personal articles in German musical lexicography.

\section{Key Words}

Felipe Pedrell - Higinio Anglés - Relations with Germanspeaking musicology - Reception of their writings in Germanspeaking countries.

\section{Resumen}

Felipe Pedrell e Higinio Anglés, ambos de orígen catalán, se vieron muy influenciados en sus estudios musicológicos por su relación con la musicología alemana. De esta manera iniciaron, por una parte, la musicología española, y por otra parte, sus conocimientos, especialmente sus ediciones y sus escritos, dieron a conocer en Alemania la música española, en particular la música medieval y renacentista. No sólo fueron valorados por amigos musicólogos sino también por su presencia continua en la lexicografía de la música alemana. [Traducción: Isolde Deleyto Rösner.].

Palabras clave

Felipe Pedrell - Higinio Anglés - Relaciones con la musicología alemana - Recepción de sus escritos en el ámbito germanófono. 
In der Wissenschaft ist den sogenannten 'Gründungsvätern' ein glückliches Nachleben sicher. Sie waren und bleiben die ersten einer bestimmten Stoßrichtung, und immer werden ihre Namen einstehen für den Beginn eines Netzwerkes von Aufschlüssen, das weiter betrieben und bis zu einem Heute vorangebracht wurde. Fehleinschätzungen verzeiht man ihnen gerne - weiß man doch, dass ihnen die Einsicht einer entwickelten akademischen Forschung noch nicht vorlag, und manchmal ist man sogar dankbar dafür, an den Angelpunkten ihrer Thesen drehen und neue Perspektiven daraus entfalten zu dürfen. Aus Forschungen im Zeichen des musikalischen Historismus heraus haben sich diese Anfänge für die Musikwissenschaft im deutschsprachigen Raum in der zweiten Hälfte des 19. Jahrhunderts ergeben und um die Jahrhundertwende eine Art 'Pilotfunktion' für zahlreiche europäische Länder zugesetzt. Am Rande erfasste diese Signalwirkung noch Felipe (Felip) Pedrell i Sabaté (1841-1922), dessen Forschergeist sich freilich aus einer 'Universalgelehrtheit' in Sachen Musik ganz im Geist des 19. Jahrhunderts erhob, umso mehr aber betraf sie dann seinen Schüler Higinio (Higini) Anglés Pamies (1888-1969), der durch ein Studium an den deutschen Universitäten Freiburg im Breisgau und Göttingen Kontakte knüpfte, die zeit seines Lebens anhielten und ihn - gemeinsam mit seiner exzellenten Laufbahn in kirchlichen Ämtern - schließlich zu einer zentralen Figur im aufkeimenden Bewusstsein einer international aufgestellten Musikwissenschaft werden ließen ${ }^{1}$.

Die Leistungen und Bedeutung von Pedrell und Anglés wurden und werden in der deutschsprachigen Musikwissenschaft durch entsprechende, durchaus ausführlichere Artikel in den beiden Auflagen der Musikenzyklopädie Die Musik in Geschichte und Gegenwart (MGG) gewürdigt, worin ihre Lebenswege ebenso wie das bleibende Vermächtnis ihrer Tätigkeiten zusammenfassend dargestellt und in der zweiten Auflage im neuesten Stand der biographischen Forschung aktualisiert worden $\operatorname{sind}^{2}$. Dagegen sind die Artikel zu den beiden Musikforschern in den zwei letzten Auflagen des knapper gefassten Riemann Musik Lexikon in Umfang und Informationsgehalt in etwa gleich geblieben ${ }^{3}$ - einesteils ein Indiz für die Solidität ihrer Arbeiten, andernteils aber auch für den unvermeidlichen Umstand, dass die Ergebnisse ihrer Forschungen allmählich durch neuere und detailliertere Studien überwachsen werden.

Spannend gestaltet sich die allerdings verzweigte Suche nach Themenfeldern, die Pedrell bzw. Anglés in der deutschsprachigen Musikwissenschaft als zentral für ihren jeweiligen Aktionsradius zugeordnet werden ${ }^{4}$. Bei Pedrell tritt in der Erst-

1 Für Näheres s. BONASTRE I BERTRAN, 17/18 (Barcelona, 2007/08): 41-65.

2 Zu Felipe Pedrell: QUEROL (1962): 989-991. BONASTRE (2005): 232-234. Zu Higinio Anglès: ANGLÈS (1949): 482. GÓMEZ (1999): 726-728.

3 Zu Felipe Pedrell: Art. Pedrell (1979): 286. Art. Pedrell (2012): 131. Zu Higinio Anglès: Art. Anglès (1978): 43. Art. Anglès (2012): 71.

4 Hierzu wurden die Registerbände der beiden Auflagen der $\mathrm{Mu}-$ sikenzyklopädie Die Musik in Geschichte und Gegenwart (MGG) he- auflage des $M G G$ insbesondere seine Bedeutung als Lehrer oder aber Vorbild sowohl von Komponisten als auch von Musikforschern in Katalonien bzw. Spanien zutage, gelegentlich wird auch sein kompositorisches Erbe angesprochen. In der Zweitauflage sind dann vermehrt auch Gattungen der Musik erwähnt, denen sich Pedrell als Komponist oder Forscher gewidmet hat, mit einem Schwerpunkt auf seine editorische Tätigkeit, die ihn unter anderem durch die Gesamtausgabe der Werke von Tomás Luís de Victoria von 1902 bis 1913 mit dem Leipziger Verlag Breitkopf \& Härtel verbunden hatte.

Vor allem Editionen, außerdem Beiträge im Rahmen der Sammelbände der Internationalen Musikgesellschaft, einige Kompositionen, musiktheoretische Schriften, die Initiativen zur Gründung von Musikzeitschriften sowie "treffliche bibliographische Kenntnisse", die sich in seinen Texten niederschlagen würden, hebt in einer Würdigung der Verdienste Pedrells um die Musikwissenschaft anlässlich dessen achtzigsten Geburtstags auch der später vor allem für sein 1919 aufgelegtes Handbuch der Notationskunde geschätzte Berliner Musikwissenschaftler Johannes Wolf hervor. Wie nahe er sich dem Jubilar fühlte, geht eindrucksvoll aus den ersten Zeilen dieses kleinen Beitrags hervor: "Am 19. Februar vollendet Felipe Pedrell sein achtzigstes Lebensjahr. Die deutsche Musikwissenschaft sendet dem Meister herzlichste Grüße und freundliche Wünsche für sein ferneres Wohlergehen. Sie fühlt sich ihm durch Methode und Ziel aufs engste verbunden. Gleichgerichtetes Streben vereinte uns vor dem Kriege [gemeint der Erste Weltkrieg, d. Verf.] in langjähriger gemeinsamer Arbeit im Bereiche der Internationalen Musikgesellschaft, und treues Festhalten am alten Plane führte uns im Fürstlichen Institut für musikwissenschaftliche Forschung in Bückeburg ${ }^{5}$, deren außerordentliches Mitglied der Jubilar ist, wieder zusammen"'

Musikwissenschafter dieses Instituts, namentlich Oskar Fleischer und Max Seiffert, suchten Pedrell für die Idee einer spanischen Sektion der "Internationalen Musikgesellschaft" zu gewinnen, zu deren Gründung es aber im Weiteren nicht $\mathrm{kam}^{7}$. Pedrell konnte allerdings - wenngleich unter manchmal schwierigen Umständen - zwischen 1900 und 1910 mehrmals umfassende Studien in den Sammelbänden der Internationalen Musikgesellschaft veröffentlichen, zudem Artikel für die fünfte Auflage des Riemann'schen Musik-Lexicon beisteuern und er

rangezogen: MGG, vol. 17, Kassel, Bärenreiter, 1986: 20, 573; $\mathrm{MGG}^{2}$, Register Personenteil, Kassel u.a. - Stuttgart / Weimar, Bärenreiter Metzler, 2007: 19, 477, sowie Register Sachteil, Kassel 1999: 275, 585. Auf den Nachweis der einzelnen Artikel, auf die sich die in den Registerbänden angeführten Referenzen beziehen, wird an dieser Stelle aus Platzgründen verzichtet.

5 Dieses Institut wurde unter fürstlicher Schirmherrschaft 1917 gegründet und war Vorläufer des 1935 ins Leben gerufenen Staatlichen Instituts für Deutsche Musikforschung in Berlin (heute: Staatliches Institut für Musikforschung Preußischer Kulturbesitz).

6 WOLF, 3 (Stuttgart, 1921): 83-85, Zitat S. 83. Beigeschlossen ein über zehn Seiten (!) langes Verzeichnis der wissenschaftlichen und künstlerischen Leistungen Pedrells: REIFF, 3 (Stuttgart, 1921): 86-97.

7 Näheres siehe BONASTRE I BERTRAN, 20/21 (Barcelona, 2013/14): 171-193. 
wurde auch eingeladen, einen Beitrag für die Festschrift zum 60. Geburtstag von Hugo Riemann bereitzustellen, die 1909 $\operatorname{erschien}^{8}$. Dass Pedrell ein "treuer Anhänger Wagners" war", wird heute kaum mehr betont ${ }^{10}$, dass er eine spanische nationale Schule der Musik initiiert hat, umso öfter. Hier fallen vor allem die Namen von Isaac Albéniz und Manuel de Falla, wie auch im Zuge der im Jahr 2000 publizierten umfassenden Studie Manuel de Falla und die Idee der spanischen Nationaloper von Eckhard Weber, der die Thematik "Falla und Pedrell" in einem ausführlichen Kapitel aufgreift ${ }^{11}$. Wertschätzung, wie sie hier begegnet, resultiert letztlich aus dem mutigen Schritt Pedrells, sich aus französischen Vorbildern und vom Magneten Paris zu lösen - und zwar sowohl in musikalischen als auch in musikhistorischen Belangen.

Bei Higinio Anglés besteht, fußend auf dessen Studium bei Wilibald Gurlitt in Freiburg im Breisgau und danach bei Friedrich Ludwig in Göttingen sowie angesichts seines Aufenthaltes in Deutschland während des Spanischen Bürgerkriegs (1936-39), als er in München bei Rudolf von Ficker und Otto Ursprung Vorlesungen hörte, auf mehreren Ebenen eine starke Bindung an die deutschsprachige Fachtradition. Einerseits prägten diese Studienjahre den Musikforscher Anglés - sie lenkten seine Aufmerksamkeit insbesondere auf spanische musikalische Quellen aus dem Mittelalter und der Renaissance, von denen er viele in Ausgaben zugänglich machte. Andererseits hatte sich Anglés die deutsche Sprache (wie im Übrigen mehrere weitere) sosehr angeeignet, dass er darin sogar zu publizieren vermochte. Etliche Aufsätze bezeugen dies, und sie lassen zugleich erkennen, wie gut Anglés mit der deutschsprachigen Musikwissenschaft der Jahre vor und nach dem Zweiten Weltkrieg vernetzt war. Mit Sicherheit trug zu dieser Akzeptanz entscheidend bei, dass Anglés als Leiter der Musikabteilung der Biblioteca de Catalunya - dazu war er 1917 berufen worden - bereits über großes Fachwissen und profunde Einblicke in das der deutschsprachigen Musikwissenschaft bis dato nahezu unbekannte 'Terrain' der iberischen Halbinsel verfügte, als er sich 1923 erstmals nach Deutschland begab.

Mit einem Anhang zur spanischen Musikgeschichte in der Übersetzung der Geschichte der Musik von Johannes Wolf ${ }^{12}$ ins Spanische, die 1934 in Barcelona erschien ${ }^{13}$, setzte Anglés nicht nur ein Repertorium der deutschsprachigen Musikwissenschaft fort, sondern nahm zugleich jene Verbindung erneut auf, die

8 Ibid., $192 f$.

9 WOLF, 3 (Stuttgart, 1921): 83. Zu den Hintergründen vgl. YOUNG, 41 (Berkeley, 2017): 31-47.

10 Eine Erwähnung Pedrells findet sich denn auch nicht in neueren Standardwerken zu Richard Wagner, weder in Daniel Brandenburg, Rainer Franke und Anno Mungen (eds.) im Auftrag des Forschungsinstituts für Musiktheater Thurnau, Das Wagner-Lexikon, Laaber, Laaber, 2012, noch in Laurenz Lütteken (ed.), Wagner Handbuch, Kassel et al. - Stuttgart / Weimar, Bärenreiter - Metzler, 2012.

11 WEBER (2000): 89-121.

12 WOLF (1925-29).

13 WOLF (1934). zwischen Pedrell und Wolf seit Längerem bestanden hatte. Von zahlreichen weiteren Kontakten zu Musikwissenschaftlern im deutschsprachigen Raum zeugen unter anderem Beiträge von Anglés' in den Festschriften für Joseph Müller-Blattau (1960), Heinrich Besseler (1961), Karl Gustav Fellerer (1962) und für den emigrierten Egon Wellesz (1966) ${ }^{14}$. Insbesondere zu Besseler, der unter Anglés' Nachfolger Miguel Querol 1949 zum "Colaborador honorario" des Instituto Español de Musicología ernannt wurde, bestand seit Anglés' Studienzeit in Deutschland eine enge Verbindung ${ }^{15}$. Gestützt wurde sie über die Aktivitäten der Internationalen Gesellschaft für Musikwissenschaft ${ }^{16}$, als deren Vizepräsident Anglés von 1933 bis 1958 fungierte. Meilensteine der Gemeinsamkeit stellten der vierte Kongress der Internationalen Gesellschaft für Musikwissenschaft in Barcelona $1933^{17}$, die - oft auf dem Umweg über den Schweizer Kollegen Jacques Handschin geführte - Korrespondenz mit Besseler zur Zeit des Nachkriegsdeutschlands ${ }^{18}$ und - nunmehr in größerem Rahmen - Anglés' tatkräftige Unterstützung der deutschen Musikwissenschaftler im Bestreben, nach Ende des Zweiten Weltkrieges wieder internationale Anerkennung zu gewinnen, dar ${ }^{19}$. Diesem Einsatz trug die Verleihung des Großen Verdienstkreuzes der Bundesrepublik Deutschland an Higinio Anglés im Jahre 1960 ehrenvoll Rechnung.

In diesem Zusammenhang darf nicht übersehen werden, dass Anglés' Berufung zum Leiter des Pontificio Istituto di Musica Sacra in Rom 1947 seiner Stimme international große Nachhaltigkeit verlieh. Zugleich waren fundierte Beiträge aus seiner nunmehr so prominenten Feder höchst gefragt, umso mehr, als Anglés sich durch die Veranstaltung großer kirchenmusikalischer Kongresse (in Rom, Wien, Paris, Köln und Chicago / Milwaukee) weiterhin profilierte. Als Autor widmete er sich zunehmend Fragen der Kirchenmusik, ohne aber sein Interesse für die 'altspanische' Musik aus den Augen zu verlieren, wie Beiträge in den Spanischen Forschungen der Görresgesellschaft und in der Geschichte der katholischen Kirchenmusik

14 Anglés, Higinio, "Die volkstümlichen Melodien bei den Trouvères", Walter Salmen (ed.), Festgabe für Joseph Müller-Blattau zum 65. Geburtstag, Saarbrücken, Universitäts- und Schulbuchverlag, 1960: 11-18; "Die Mehrstimmigkeit des Calixtinus von Compostela und seine Rhythmik", Eberhard Klemm (ed.), Festschrift Heinrich Besseler zum sechzigsten Geburtstag, Leipzig, VEB Deutscher Verlag für Musik, 1961: 91-100; "Die Bedeutung der Plika in der mittelalterlichen Musik", Heinrich Hüschen (ed.), Festschrift Karl Gustav Fellerer zum sechzigsten Geburtstag, Regensburg, Bosse, 1962: 28-39; "Sakraler Gesang und Musik in den Schriften Gregors des Großen", Alan Jack Westrup (ed.), Essays presented to Egon Wellesz, Oxford, Clarendon, 1966: 33-42.

15 Näheres s. BÄCKER, 61 (Barcelona, 2006): 273-295.

16 Nachfolgeinstitution der Internationalen Musikgesellschaft, die de facto schon seit 1914, ausgelöst durch den Ersten Weltkrieg, nicht mehr existiert hatte. Siehe http://www.musiklexikon.ac.at/ml/musik_I/ IMG.xml (zuletzt aufgerufen 18.09.2013).

17 Dazu siehe insbesondere CALMELL i PIGUILLEM, 70 (Barcelona, 2015): 161-178.

18 BÄCKER, 61 (Barcelona, 2006): 276 bzw. 274f.

19 FELLERER, 23 (Kassel, 1970): 125. 
zeigen $^{20}$. Die Solidität seiner wissenschaftlichen Leistungen spiegelt sich schließlich in der Namhaftigkeit der deutschsprachigen Autoren, die zu den Miscelánea en homenaje a Monseñor Higinio Anglés beitrugen ${ }^{21}$. Unter anderem finden sich darin Texte von Hans Joachim Moser, Joseph Müller-Blattau, Leopold Nowak, Helmuth Osthoff, Walter Salmen, Arnold Schmitz, Marius Schneider, Leo Schrade und Bruno Stäblein. Dass manche der Musikwissenschaftler, die mit Anglés in Kontakt standen, durchaus mit dem nationalsozialistischen Régime kollaboriert hatten (in erster Linie Müller-Blattau, aber auch Besseler) ${ }^{22}$, wog damals nicht schwer oder war noch gar nicht weiter bekannt geworden ${ }^{23}$.

Higinio Anglés ist es zu verdanken, dass - nicht zuletzt auf der Grundlage seiner an deutschen Universitäten vervollkommneten wissenschaftlichen Arbeitstechnik - die spanische Musik des Mittelalters und der Renaissance durch sorgfältige musikalische Ausgaben und gründlich recherchierte Aufsätze einem breiten Kreis bekannt gemacht wurde. Exemplarisch verdeutlicht die Studie "Die Cantigas de Santa Maria König Alfons' des Weisen”, mit der er postum 1973 zur Gedenkschrift Leo Schrade beitrug ${ }^{24}$, welche Einsichten Anglés aus dem Studium der Quellen heraus in Bezug auf Form, Rhythmus und musikgeschichtliche Verbindungslinien zu erschließen vermochte. Sein Interesse für Volksmusik - schon in der Jugend hatte er katalanische Volkslieder gesammelt, und von Pedrell wurde er in deren Wertschätzung bestätigt - schärfte den Blick für den musikalischen Austausch zwischen Gregorianik und volksläufiger Überlieferung, vergleichbar den Studien des ungarischen Musikwissenschaftlers Benjamin Rajeczky (1901-1981) ${ }^{25}$; der

20 Anglès, Higinio, "Die Rolle Spaniens in der mittelalterlichen Musikgeschichte", Johannes Vincke (ed.), Gesammelte Aufsätze zur Kulturgeschichte Spaniens, vol. 19/20, Münster, Aschendorff, 1962 (Spanische Forschungen der Görresgesellschaft, 19/20): 1-24; "Un tractat de cant plà d'autor anònim del segle XVI”, Johannes Vincke (ed.), Gesammelte Aufsätze zur Kulturgeschichte Spaniens, vol. 21, Münster, Aschendorff, 1963 (Spanische Forschungen der Görresgesellschaft, 21): 277-293; "Spanisch-mozarabische Liturgie", Karl Gustav Fellerer (ed.), Geschichte der katholischen Kirchenmusik, vol. 1: Von den Anfängen bis zum Tridentinum, Kassel u.a., Bärenreiter, 1972: 208-219. Siehe auch die in Anmerkung 14 genannten, in diversen Festschriften publizierten Aufsätze.

21 CONSEJO SUPERIOR DE INVESTIGACIONES CIENTÍFICAS (1958/61).

22 Vgl. SCHULER (2006), 319-327. SCHIPPERGES (2006).

23 Anglès, der nach der Machtübernahme General Francisco Francos zum ersten Leiter der Instituto Español de Musicología berufen worden war, zeichnete schon damals eine grundsätzlich offene Einstellung gegenüber seiner Kollegenschaft im Fach aus, indem er seinerzeit mit emigrierten Kollegen wie dem Musikkritiker Adolfo Salazar und dem Komponisten Roberto Gerhard weiterhin Kontakt pflegte; siehe dazu MOREDA RODRÍGUEZ, 96 (Oxford, 2015), 209-227.

24 Anglès, Higinio, "Die Cantigas de Santa Maria König Alfons' des Weisen", Wulf Arlt, Ernst Lichtenhahn und Hans Oesch (eds.), Gattungen der Musik in Einzeldarstellungen. Gedenkschrift Leo Schrade, Bern / München, Francke, 1973: 346-364.

25 Vgl. dazu unter anderem Anglès, Higinio, "Die Bedeutung des Volksliedes für die Musikgeschichte Europas", Wilfried Brennecke, deutschsprachigen Fachkollegenschaft wurden dadurch Wege für die Zukunft gewiesen.

Als Leiter des Pontificio Istituto di Musica Sacra nahm Anglés dagegen eine traditionsverhaftete Position ein, die ihn während des Zweiten Vatikanischen Konzils mehr und mehr zum Rufer in der Wüste werden ließ. Eine Neigung zu rigoroser Wertung, die Anglés generell attestiert wird ${ }^{26}$, steigerte sich ins Polemische. "Mit großer Sorge verfolgte er [...] die Bestrebungen amusischer Rationalisten, den Raum der Kirchenmusik als Kunst im katholischen Gottesdienst einzuengen und sie durch fragwürdige musikalische Gebilde zu ersetzen. Seine letzten Lebensjahre waren vom Kampf um sein kirchenmusikalisches Ideal erfüllt, reich an Enttäuschungen auch durch Menschen, denen er sein volles Vertrauen geschenkt hatte"27.

Anglés' Haltung - die sich letztlich nicht durchsetzen konnte - war geprägt von musikhistorischer Verwurzelung. Mit Verweis auf die restaurativen Tendenzen des 19. Jahrhunderts hatte er eingemahnt, dass kirchenmusikalische Ideale überzeitliche Geltung beanspruchen würden und diese namentlich im Motu proprio "Tra le sollecitudini” Pius' X. von 1903 festgeschrieben seien ${ }^{28}$. Die Zukunft der Kirchenmusik lag für ihn in einer vermehrten Toleranz gegenüber dem musikalischen Erbe unter Beibehaltung der lateinischen Missa cantata. "Es besteht auf dem Gebiet der Liturgie sowie auf dem der Sakralmusik ein anarchistischer Zustand, zugunsten einer 'seelsorglichen Liturgie' wird die feierliche Liturgie samt ihrem Latein und auch die wahrhaft künstlerische Sakralmusik geopfert"29. Sich mit solchen Stellungnahmen in den konziliaren Diskurs einzuschalten, musste zwangsläufig zu einer Isolierung Anglés' führen. Dass dies aber den Wert seiner sonstigen musikologischen Schriften nicht entscheidend schmälerte, beweist deren Resonanz bis zum heutigen Tag.

Anglés' Wirken wird 1965 im Artikel "Spanien" in der Erstauflage des $M G G$ umfassend gewürdigt, wenn es heißt: "Die Musikwissenschaft hat ihren vorzüglichsten Vertreter in H. Anglés. In dem staatlichen Institut, dessen Leitung ihm übertragen wurde [gemeint ist das 1943 gegründete Instituto Español de Musicología, d. Verf.], arbeiten M. Querol Gavaldà, F. Baldelló, E. Pujol, S. Kastner, der Folklorist Manuel García Matos und J. Subirà. Auf diese Weise hat die umfassende Arbeit Pedrells

Willi Kahl und Rudolf Steglich (eds.), Bericht über den Internationalen musikwissenschaftlichen Kongress Bamberg 1953, Kassel / Basel, Bärenreiter, 1954: 181-184.Zu Rajeczky vgl. Suppan, Wolfgang, Volkslied. Seine Sammlung und Erforschung, Stuttgart, Metzler, ${ }^{2} 1978$ (Sammlung Metzler. Realien zur Literatur, Abt. E: Poetik, 52): 2, sowie Tari, Lujza, "Benjamin Rajeczky the Ethnomusicologist", Studia musicologica Academiae Scientiarum Hungaricae, 42 (Budapest, 2001), 3/4: 213-252.

26 GÓMEZ (1999): 727.

27 FELLERER, 23 (Kassel, 1970): 126.

28 Anglès, Higinio, "Das kommende Ökumenische Konzil und die Kirchenmusik" [übersetzter Nachdruck eines Beitrags im Bollettino degli amici del Pontificio Istituto di Musica Sacra], Singende Kirche, 8 (Wien, 1960): 150-156.

29 Ibid., 154. 
eine bemerkenswerte Fortsetzung erfahren"30 . Besonders geschätzt werden - wie eine Durchsicht der $M G G$-Bände beider Auflagen ergibt - einige grundlegende Publikationen Anglés', darunter das gemeinsam mit Joaquín Pena und Miguel Querol Gavaldà herausgegebene Diccionario de la Música von 1954, die Studien zur mittelalterlichen Musik Spaniens sowie seine verschiedentlichen Editionen. Higinio Anglés hat damit Grundlagen geschaffen, an denen auch die deutschsprachige Musikwissenschaft, sofern sie sich der Musikgeschichte Spaniens zuwendet, nicht vorbeigehen konnte und kann - wenn sie auch im Wesentlichen Quellen und Entwicklungen bis zu Beginn des 16. Jahrhunderts betrafen, als die Habsburger die Regentschaft über das spanische Königreich übernahmen.

\section{BIBLIOGRAPHIE}

Art. "Anglès, Higini", Carl Dahlhaus / Hans Heinrich Eggebrecht (eds.), Brockhaus Riemann Musiklexikon, Bd. 1, Wiesbaden / Mainz, Brockhaus / Schott 1978, 43.

Art. "Anglès, Higini”, Wolfgang Ruf coll. Annette van DyckHemming (ed.), Riemann Musik Lexikon, Bd. 1, Mainz, Schott, 2012, 71.

Art. "Pedrell, Felipe", Carl Dahlhaus / Hans Heinrich Eggebrecht (eds.), Brockhaus Riemann Musiklexikon, Bd.2, Wiesbaden / Mainz, Brockhaus / Schott, 1979, 286.

Art. "Pedrell, Felipe", Wolfgang Ruf coll. Annette van DyckHemming (ed.), Riemann Musik Lexikon, Bd. 4, Mainz, Schott, 2012, 131.

Anglés, Higinio, Art. “Anglés, Higino”, Friedrich Blume (ed.), Die Musik in Geschichte und Gegenwart, Bd. 1, Kassel / Basel, Bärenreiter, 1949, 482.

Bäcker, Rolf, "Relaciones internacionales del Instituto Español de Musicología durante los primeros años del trabajo de Miguel Querol: El ejemplo de Heinrich Besseler", Anuario musical, 61 (Barcelona, 2006), 273-295.

Bonastre [i Bertran], Francesc, Art. "Pedrell i Sabaté, Felipe/ Felip”, Ludwig Finscher (ed.), Die Musik in Geschichte und Gegenwart, 2. Auflage, Personenteil Bd. 13, Kassel et al. - Stuttgart / Weimar, Bärenreiter - Metzler, 2005, 232-234.

Bonastre i Bertran, Francesc, "Els orígens del concepte de barroc musical hispànic, a l'entorn del centenari de l'Institut d'Estudis Catalans: Felip Pedrell, Higini Anglès, Miquel Querol", Recerca Musicòlogica, 17/18 (Barcelona, 2007/08), 41-65.

Bonastre i Bertran, Francesc, "La presencia de Felip Pedrell en el proyecto fundacional de la Sociedad Internacional de Musicología (1899)", Recerca musicològica, 20/21 (Barcelona, 2013/14), 171-193.
Calmell i Piguillem, César, "El III Congreso Internacional de Musicología en Barcelona 1936, a partir de la documentación guardada en el Fondo Higini Anglés de la Biblioteca de Catalunya", Anuario musical, 70 (Barcelona, 2015), 161-178.

Consejo Superior de Investigaciones Científicas (ed.), Miscelánea en homenaje a Monseñor Higinio Anglés. 2 vol., Barcelona, Consejo Superior de Investigaciones Científicas, $1958 / 61$.

Fellerer, Karl Gustav, "Higino Anglés †", Die Musikforschung, 23 (Kassel, 1970), 125-127.

Gómez, Maricarmen, Art. “Anglés, Higino”, Ludwig Finscher (ed.), Die Musik in Geschichte und Gegenwart, 2. Auflage, Personenteil Bd. 1, Kassel et al. - Stuttgart / Weimar, Bärenreiter - Metzler, 1999, 726-728.

Moreda Rodríguez, Eva, "Early Music in Francoist Spain”, $M u$ sic \& Letters, 96 (Oxford, 2015), 209-227.

Querol, Miguel, Art. "Pedrell, Felipe", Friedrich Blume (ed.), Die Musik in Geschichte und Gegenwart, Bd. 10, Kassel et al., Bärenreiter, 1962, 989-991.

Reiff, Alfred, "Ein Katalog zu den Werken von Felipe Pedrell", Archiv für Musikwissenschaft, 3 (Stuttgart, 1921), 86-97.

Schipperges, Thomas, Die Akte Heinrich Besseler. Musikwissenschaft und Wissenschaftspolitik in Deutschland 1924 bis 1949. München, Strube, 2005 (Quellen und Studien zur Musik in Baden-Württemberg 7).

Schuler, Manfred, "Zum völkisch-nationalen Denken in der deutschen Musikwissenschaft", Isolde von Förster / Christoph Hust (eds.), Musikforschung - Faschismus Nationalsozialismus. Referate der Tagung Schoß Engers (8. bis 11. März 2000), Mainz, Are Musik-Verlag, 2001, 319-327.

Subira, José, "VIII. 18. bis 20. Jahrhundert" im Art. "Spanien”, Friedrich Blume (ed.), Die Musik in Geschichte und Gegenwart, Bd. 12, Kassel et al., Bärenreiter, 1965, 10041010: 1010 .

Weber, Eckhard, Manuel de Falla und die Idee der spanischen Nationaloper. Diss. Freie Universität Berlin 1998, Frankfurt a. M. et al., Lang, 2000 (Perspektiven der Opernforschung, 7).

Wolf, Johannes, "Felipe Pedrell zum achtzigsten Geburtstage", Archiv für Musikwissenschaft, 3 (Stuttgart, 1921), 83-85.

Wolf, Johannes, Geschichte der Musik in allgemeinverständlicher Form. 3 Teile, Leipzig 1925-29, ${ }^{2} 1930-35$.

Wolf, Johannes, Historia de la Música. Con un estudio crítico de Historia de la Música española. Übersetzt von Roberto Gerhard, Barcelona 1934.

Young, Clinton D., "The Southern Slope of Monsalvat: How Spanish Wagnerism Became Catalan", 19th Century Music, 41 (Berkeley, 2017), 31-47.
Recibido: 29.03.2018

Aceptado: 16.04.2018

30 SUBIRA (1965): 1010. 